\title{
Interactions between Neutrophils and Leishmania braziliensis Amastigotes Facilitate Cell Activation and Parasite Clearance
}

\author{
Eric D. Carlsen ${ }^{a, b}$ Zuliang Jie ${ }^{b} \quad$ Yuejin Liang $^{b}$ Calvin A. Henard ${ }^{b}$ Christie Hay ${ }^{b}$ \\ Jiaren Sun ${ }^{b}$ Herbert de Matos Guedes ${ }^{c}$ Lynn Soong ${ }^{b}$ \\ ${ }^{a} \mathrm{MD}-\mathrm{PhD}$ Combined Degree Program and ${ }^{\mathrm{b}}$ Department of Microbiology and Immunology, University of Texas \\ Medical Branch, Galveston, Tex., USA; ' Laboratório de Inflamação, Instituto de Biofísica Carlos Chagas Filho, \\ Universidade Federal do Rio de Janeiro, Polo avançado de Xerém, Rio de Janeiro, Brazil
}

\section{Key Words}

Leishmania $\cdot$ Neutrophil $\cdot$ Macrophage $\cdot$ Reactive oxygen

species · Degranulation · Interleukin 22

\begin{abstract}
Leishmania braziliensis and Leishmania amazonensis are both causative agents of cutaneous leishmaniasis in South America. However, patient prognosis and the host immune response differ considerably depending on the infecting parasite species. The mechanisms underlying these differences appear to be multifactorial, with both host and parasite components contributing to disease outcome. As neutrophils are a prominent component of the inflammatory infiltrate in chronic cutaneous, diffuse cutaneous and mucocutaneous lesions, we examined neutrophil activation and microbicidal activity against amastigotes of $L$. amazonensis and $L$. braziliensis. We found that murine neutrophils internalized $L$. braziliensis amastigotes with greater efficiency than did L. amazonensis amastigotes. Additionally, L. braziliensis infection was a potent trigger for neutrophil activation, oxidative burst, degranulation and the production of interleukin (IL)-22 and IL-10, while L. amazonensis amastigotes poorly induced these responses. Finally, neutrophils were able to kill $L$. braziliensis amastigotes, especially when cells were activated with phorbol myristate acetate. L. amazonensis amastigotes, however, were highly resistant to neutrophil
\end{abstract}

microbicidal mechanisms. This study reveals, for the first time, differential neutrophil responsiveness to distinct species of Leishmania amastigotes and highlights the complexity of neutrophil-amastigote interactions during chronic leishmaniasis.

(c) 2015 S. Karger AG, Basel

\section{Introduction}

Leishmania braziliensis and L. amazonensis are causative agents of cutaneous leishmaniasis in overlapping endemic areas of South America. Infection by these two species causes immunologically distinct forms of secondary leishmaniasis in patients, resulting in contrasting host immune response profiles and disease outcomes. Cellular hypersensitivity against $L$. braziliensis can induce mucocutaneous leishmaniasis, which is characterized by extensive nasopharyngeal tissue destruction [1]. In contrast, cellular hyposensitivity against L. amazonensis can result in diffuse cutaneous leishmaniasis, which is characterized by uncontrolled parasite dissemination throughout the skin [2]. Currently, the mechanisms responsible for the opposing immune responses elicited by these two parasite species (particularly against the amastigote parasite stage that causes disease in mammals) remain poorly defined.

\section{KARGER 125}

2015 S. Karger AG, Base

$1662-811 \mathrm{X} / 15 / 0074-0354 \$ 39.50 / 0$

E-Mail karger@karger.com

www.karger.com/jin
Dr. Lynn Soong

Department of Microbiology and Immunology, University of Texas Medical Branch

Medical Research Building 3.132, 301 University Blvd

Galveston, TX 77555-1070 (USA)

E-Mail lysoong@utmb.edu 
We and other study groups have demonstrated that amastigotes of $L$. braziliensis and L. amazonensis differ considerably in their ability to induce innate immune cell functions in vitro. For example, infection of murine bone marrow-derived dendritic cells (DC) with $L$. braziliensis amastigotes results in the potent upregulation of CD40 and interleukin (IL)-12 release [3], while DC infection with $L$. amazonensis amastigotes results in poor expression of CD40, CD83 and IL-12 [4]. Similarly, L. braziliensis amastigotes potently trigger human monocyte oxidative burst, resulting in reactive oxygen species (ROS)-dependent parasite clearance [5] whereas monocytes infected with L. amazonensis amastigotes are defective in oxidative burst [6]. To better understand amastigote interactions with other innate immune cells, we recently examined neutrophil activation and microbicidal activity against $L$. amazonensis amastigotes. We found that amastigotes are a poor stimulus for neutrophil activation and are highly resistant to neutrophil microbicidal activity [7]. These findings supported an earlier report from our laboratory that $L$. amazonensis amastigotes are not killed by purified human histones [8], which are important microbicidal components of neutrophil extracellular traps (NETs).

Currently, little is known about neutrophil interactions with $L$. braziliensis amastigotes, despite a recent report that neutrophils are a prevalent component of the inflammatory infiltrate in mucocutaneous leishmaniasis [9]. In this study, we examined the ability of L. braziliensis amastigotes to trigger neutrophil activation, and tested whether they are susceptible to neutrophil microbicidal mechanisms. We found that, in comparison to L. amazonensis, L. braziliensis amastigotes were more efficiently internalized by neutrophils and induced significantly more neutrophil activation, oxidative burst, degranulation and cytokine production. Potent neutrophil activation by L. braziliensis amastigotes was associated with efficient parasite clearance by these cells, especially in the presence of phorbol myristate acetate (PMA). L. amazonensis amastigotes, however, were highly resistant to killing by neutrophils, even when cocultured with PMA-activated cells. Interestingly, we observed that uninfected neutrophils can enhance the clearance of both species through favorable interactions with infected macrophages. Our results suggest that L. amazonensis and $L$. braziliensis amastigotes differ considerably in their ability to trigger neutrophil activation and degranulation, and that these differences correspond with the capacity of neutrophils to directly clear these parasites. The finding that neutrophils can contribute to amastigote control (ei- ther directly or by enhancing macrophage microbicidal activity) highlights a potential role for these cells in limiting parasite growth in chronic cases of leishmaniasis.

\section{Materials and Methods}

Mice and Reagents

Female C57BL/6 and BALB/c mice were purchased from Taconic Farms (Germantown, N.Y., USA). Syrian Golden hamsters were purchased from Harlan Sprague Dawley (Indianapolis, Ind., USA). All animals were maintained under specific pathogen-free conditions and used in accordance with protocols approved by the Animal Care and Use Committee of the University of Texas Medical Branch (Galveston, Tex., USA). All chemical reagents were purchased from Sigma Aldrich (St. Louis, Mo., USA) unless otherwise specified.

\section{Parasite Cultivation}

Infectivity of L. amazonensis (strain RAT/BA/74/LV78) and $L$. braziliensis (strain LC1418) was maintained by regular passage through BALB/c mice and Syrian Golden hamsters, respectively. Promastigotes of both species were cultured at $26^{\circ} \mathrm{C}$ in Schneider's Drosophila medium (Crescent Chemical Company, Islandia, N.Y., USA), pH 7.0, supplemented with $20 \%$ heat-inactivated fetal bovine serum and gentamicin $(50 \mu \mathrm{g} / \mathrm{ml})$. Axenic amastigotes of both species were generated by culturing stationary-phase promastigotes at $32{ }^{\circ} \mathrm{C}$ in Grace's insect medium (Invitrogen, Carlsbad, Calif., USA), pH 5.2, supplemented with $20 \%$ heat-inactivated FBS and gentamicin $(25 \mu \mathrm{g} / \mathrm{ml})$, as described previously [3].

\section{Neutrophil Collection}

Peritoneal exudate cells were obtained from mice $5 \mathrm{~h}$ after injection with $3 \%$ thioglycollate broth. Thioglycollate was removed, and neutrophils were purified via density gradient centrifugation with Percoll. Neutrophil purity (approx. 95\%) was routinely assessed by fluorescence-activated cell sorting (FACS) and examination of morphology; cell viability was routinely $>95 \%$ as monitored by Trypan blue exclusion. All neutrophil experiments were carried out in tissue-culture-treated polystyrene. All neutrophil-parasite cocultures were carried out at $32^{\circ} \mathrm{C}$ with $5 \% \mathrm{CO}_{2}$.

\section{Quantifying Parasite Uptake by Neutrophils}

Leishmania amastigotes were labeled with $1 \mu \mathrm{M}$ carboxyfluorescein succinimidyl ester (CFSE) in PBS for 15 min at room temperature, followed by quenching in complete media and 2 additional washes in PBS. CFSE-labeled parasites were cocultured with neutrophils at a multiplicity of infection (MOI) of 5 for $4 \mathrm{~h}$, as described previously [7]. Cells were washed, blocked with antiCD16/CD32, and then stained with anti-Ly6G-AlexaFluor 647 (BioLegend, San Diego, Calif., USA) and anti-CD11b-PE-Cy7 (BD Bioscience, San Jose, Calif., USA). Cells were analyzed by using a LSR II Fortessa FACS (BD Bioscience). Neutrophils were identified based on forward/side scatter characteristics and Ly6G/CD11b positivity, and cell infection was identified by neutrophil acquisition of CFSE. Parasite uptake was further characterized by using an ImageStream X Mark II imaging flow cytometer (Amnis Corp., Seattle, Wash., USA) to count the number of parasites internalized by neutrophils. Images obtained from the imaging flow cytometer were captured at $\times 60$ magnification. 
Assessment of Neutrophil Activation and Oxidative Burst

Neutrophil activation was assessed by surface upregulation of CD11b, which was examined after cells were blocked with antiCD16/CD32 and stained with anti-Ly6G-APC (BD Bioscience) and anti-CD11b-PerCP-Cy5.5 (eBioscience, San Diego, Calif., USA). Oxidative burst was assessed by staining cells with antiLy6G-APC and dihydrorhodamine 123 (DHR 123, $1 \mu \mathrm{M}$ ), which converts to the fluorescent product rhodamine 123 (Rho 123) upon oxidation. The oxidation reaction was stopped on ice, and neutrophil oxidative burst was quantified by gating on Ly6G+ cells and measuring the mean fluorescence intensity (MFI) of Rho 123 by FACS. Data were collected by using an Accuri C6 flow cytometer (Accuri Cytometers Inc., Ann Arbor, Mich., USA). Activation and oxidative burst flow cytometry data were analyzed by using CFlow version 1.0.227.4 (Accuri Cytometers Inc.) and FlowJo version 7.6.1 (Tree Star, Ashland, Oreg., USA).

\section{Measurement of Neutrophil Degranulation}

Flow cytometric assessment of neutrophil degranulation was performed by measuring the extent of myeloperoxidase (MPO) loss from the cell interior. MPO values for samples are displayed as a percentage of the MFI of MPO compared to the MPO intensity of control neutrophils. Amastigote-induced degranulation was assessed after neutrophils were cocultured with parasites for $4 \mathrm{~h}$, followed by cell staining with anti-Ly6G-APC, anti-CD11b-PerCPCy5.5 and anti-MPO-FITC (Abcam, Cambridge, UK). In some experiments, amastigote-infected neutrophils also received LPS (100 $\mathrm{ng} / \mathrm{ml}$ ). As a positive control for degranulation, neutrophils were pretreated with cytochalasin B $(5 \mu \mathrm{g} / \mathrm{ml})$ for $10 \mathrm{~min}$ followed by fMLP $(10 \mu \mathrm{M})$, as described previously [10]. Corresponding MPO activity in culture supernatants from positive control experiments was assessed by adding tetramethylbenzidine and measuring optical density values at $450 \mathrm{~nm}$ on a Multiskan Ascent ELSA reader (Labsystems, Helsinki, Finland), as previously described [11].

\section{Quantifying Parasite Clearance by Neutrophils}

To ensure efficient parasite internalization, amastigotes were cocultured with a surplus of neutrophils at a ratio of 1 parasite per 10 neutrophils (MOI of 0.1 ) for $6 \mathrm{~h}$. In some experiments, cocultures were incubated with PMA $(100 \mathrm{nM})$ in the presence or absence of DNase 1 (100 U/ml, Thermo Scientific, Waltham, Mass., USA). At the end of incubation, cocultures were adjusted to $5 \mathrm{~mm}$ EDTA and incubated for $30 \mathrm{~min}$ to liberate adherent cells. Subsequently, parasite survival was assessed by limiting dilution in Schneider's Drosophila medium, as previously described [5].

\section{Macrophage-Neutrophil Cocultures}

Bone marrow-derived macrophages were generated from C57BL/6 mice (6-8 weeks old), as described previously [8]. Briefly, bone marrow cells were flushed from the mice femurs and cultured in Iscove's modified Dulbecco's medium (Invitrogen) supplemented with $10 \% \mathrm{FBS}, 1 \mathrm{mM}$ sodium pyruvate, $50 \mu \mathrm{M} 2$-mercaptoethanol, $50 \mu \mathrm{g} / \mathrm{ml}$ gentamicin, $100 \mathrm{U} / \mathrm{ml}$ penicillin and $20 \mathrm{ng} / \mathrm{ml}$ rM-CSF (recombinant murine macrophage colony-stimulating factor; eBioscience) for 10 days at $37^{\circ} \mathrm{C}$ and $5 \% \mathrm{CO}_{2}$. Cells received fresh medium containing $\mathrm{rM}-\mathrm{CSF}$ on day 5 , and were collected for use on day 10. Macrophages were infected with amastigotes of L. amazonensis or L. braziliensis at a ratio of 3:1 for $24 \mathrm{~h}$. Infected macrophages were washed to remove free amastigotes, and then cocultured with neutrophils (at a 3:1 neutrophil-to-macrophage ratio) in fresh Iscove's modified Dulbecco's medium for an additional $24 \mathrm{~h}$. At the end of the macrophage-neutrophil coculture, this medium was replaced with complete Schneider's medium and incubated at $26^{\circ} \mathrm{C}$ to facilitate amastigote conversion into promastigotes and the release of parasites from the macrophages. Live promastigotes were counted on a hemacytometer after incubating for 3-4 days.

\section{Assessing Cytokine Release in Neutrophil-Amastigote} Cocultures

Neutrophils were cocultured with amastigotes (MOI of 5) for $24 \mathrm{~h}$ in the presence of aprotinin $(50 \mu \mathrm{g} / \mathrm{ml})$ to minimize proteasemediated cytokine degradation. Cytokine levels in culture supernatants were assessed by multiplex (eBioscience) and analyzed with a Bio-Plex 200 multiplex reader equipped with Bio-Plex Manager 6.0 software (Bio-Rad Laboratories, Hercules, Calif., USA). To confirm neutrophil production of IL-22, cells were collected after $4 \mathrm{~h}$ of coculture with parasites (MOI of 5), stained for antiLy6G-AlexaFluor 647, anti-CD11b-PE-Cy7 and anti-IL-22-PE (eBioscience), and then analyzed by flow cytometry.

\section{Statistical Analysis}

Differences between the 2 groups were determined by twotailed Student's t test. Graphs were prepared by using GraphPad Prism version 4.0 (GraphPad Software, San Diego, Calif., USA). The differences between groups were considered significant when the $\mathrm{p}$ value was $<0.05$.

\section{Results}

\section{Neutrophils Competently Engulf L. braziliensis Amastigotes}

By using a combination of conventional FACS and imaging flow cytometry, we compared the ability of neutrophils to engulf CFSE-labeled amastigotes of L. amazonensis and L. braziliensis. Neutrophils were cocultured with CFSE-labeled parasites at a ratio of 5 parasites/ 1 neutrophil to ensure sufficient cell saturation. After $4 \mathrm{~h}$ of coculture, we observed that L. braziliensis uptake by CD11b+Ly6G+ neutrophils was 7 times more efficient than that of L. amazonensis (fig. 1a, $84.7 \%$ of L. braziliensis-exposed cells vs. $11.7 \%$ of L. amazonensis-exposed cells). Imaging flow cytometric analysis enabled us to compare the number of internalized parasites per infected cell, and to exclude potentially confounding anomalies such as extracellular parasite-neutrophil doublets (fig. 1b, left-most panel). We observed that nearly $70 \%$ of L. braziliensis-infected cells carried only 1 parasite, while approximately $60 \%$ of L. amazonensis-infected cells carried multiple parasites (fig. 1c). These findings demonstrate that murine neutrophils differ substantially in their ability to internalize amastigotes of $L$. amazonensis and L. braziliensis. 
Fig. 1. Neutrophil uptake of L. amazonensis and L. braziliensis amastigotes. Thioglycollate-induced peritoneal neutrophils were cocultured with CFSE-labeled amastigotes (MOI of 5) for $4 \mathrm{~h}$. Cells were then analyzed by both FACS (a) and imaging flow cytometry $(\mathbf{b}, \mathbf{c})$ to assess parasite uptake. a A histogram shows CD11b+ Ly6G+ neutrophil uptake of CFSE-labeled parasites. b Representative events from imaging flow cytometric analysis showing (leftright) an uninfected neutrophil (red) in close contact with an amastigote (green) and neutrophils infected with 1, 2 and 3 parasites, respectively. c Graphic representation of imaging flow cytometry, showing the percentage of infected cells carrying the designated number of L. amazonensis or L. braziliensis parasites. $\mathrm{La}=$ L. amazonensis-infected neutrophils (solid gray line); $\mathrm{Lb}=$ L. braziliensis-infected neutrophils (solid black line); Med = uninfected neutrophils (dotted line).

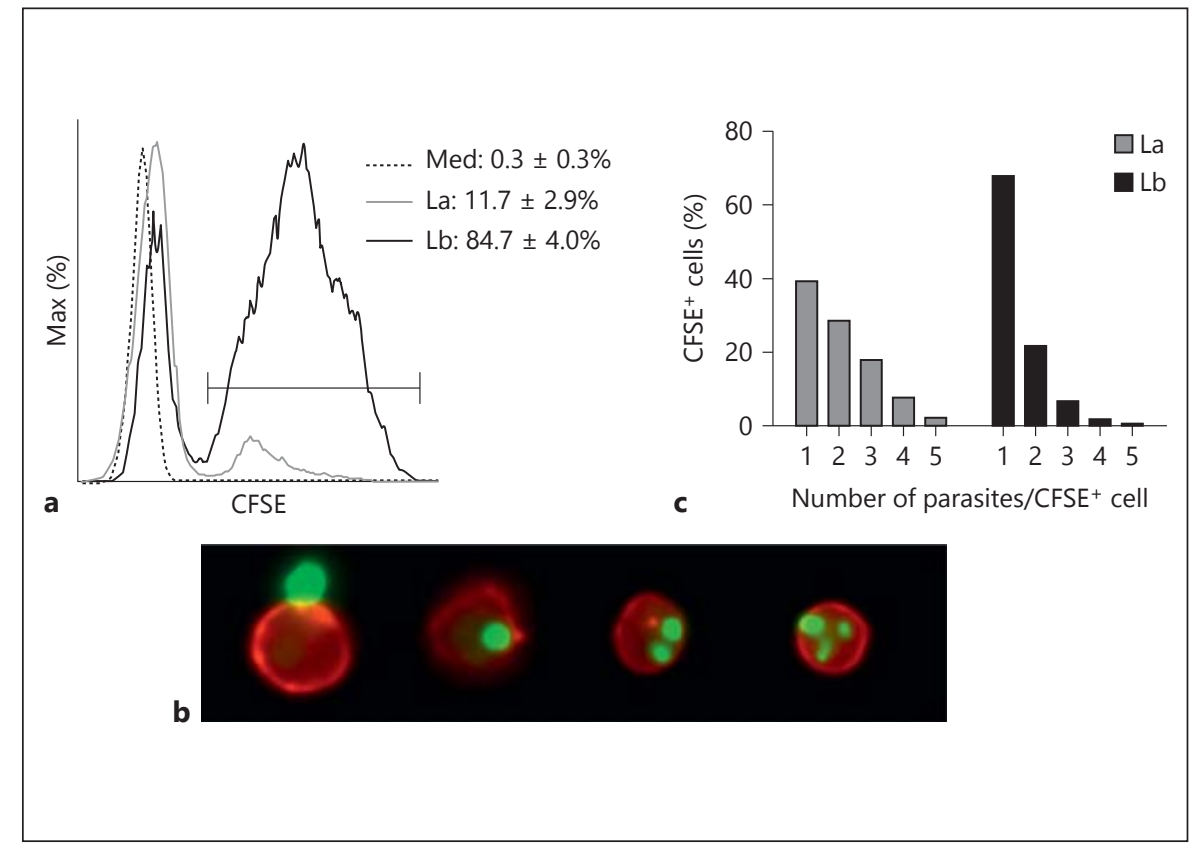

\section{L. braziliensis Amastigotes Potently Induce Neutrophil} Activation and Oxidative Burst

Surface CD11b upregulation on neutrophils is typically associated with an activated phenotype [12]. As in our previous report [7], we noted a significant increase in neutrophil surface CD11b expression after exposure to L. amazonensis amastigotes (fig. $2 \mathrm{a}$, gray bar; $\mathrm{p}<0.05$ ). Notably, L. braziliensis was a more potent trigger for CD11b exposure than L. amazonensis (fig. 2a, black bar; $\mathrm{p}<0.001$ ). L. braziliensis amastigotes also induced more CD11b expression than L. braziliensis metacyclic promastigotes (online suppl. fig. S1; for all online suppl. material, see www.karger.com/doi/10.1159/000373923). We have previously described the superior stimulatory capacity of L. braziliensis amastigotes when interacting with murine DC, as amastigotes were better able to induce IL12 production from infected cells than were their promastigote counterparts [3].

Imaging flow cytometric analysis using CFSE-labeled parasites further revealed that the extent of CD11b upregulation corresponded with the number of internalized parasites for both parasite species (fig. $2 \mathrm{~b}, \mathrm{c}$ - cells carrying 1 parasite vs. cells carrying $\geq 3$ parasites). We also noted that the level of CD11b surface expression in CFSE-neutrophils (the 0 parasite group) was higher than medium control neutrophils, implying that bystander cells are also activated in neutrophil-amastigote cocultures. Bystander activation was greater in cocultures containing L. brazil- iensis, as the CD11b MFI of these cells was approximately 2 -fold higher than that of the bystander cells in L. amazonensis cocultures. These results indicate that amastigotes can facilitate neutrophil activation in both infected and uninfected cells, with neutrophils carrying multiple parasites exhibiting the most activated phenotype.

In addition to CD11b upregulation, activated neutrophils typically undergo an NADPH-oxidase-dependent respiratory burst, resulting in the release of noxious ROS [13]. To evaluate neutrophil oxidative burst after parasite infection, we treated cells with DHR 123, a cell-permeable probe that converts into fluorescent Rho 123 upon oxidation [14]. As reported previously [7], we detected an appreciable oxidative burst response in L. amazonensis-infected cells. However, L. braziliensis infection was a significantly more potent stimulus for neutrophil ROS production (fig. 2d). By gating on Rho 123+ (ROS-producing) neutrophils and comparing the intensity of neutrophil oxidative burst on a per-cell basis, we found that the mean Rho 123 intensity in L. braziliensis-infected cells was nearly twice that of L. amazonensis-infected neutrophils (fig. 2e). Curiously, oxidative burst in response to L. braziliensis metacyclic promastigotes was much weaker than L. braziliensis amastigote-mediated burst (online suppl. fig. S2). These data collectively lead us to suggest that $L$. braziliensis amastigotes are a more effective trigger for neutrophil activation and oxidative burst than are $L$. amazonensis amastigotes or L. braziliensis promastigotes. 


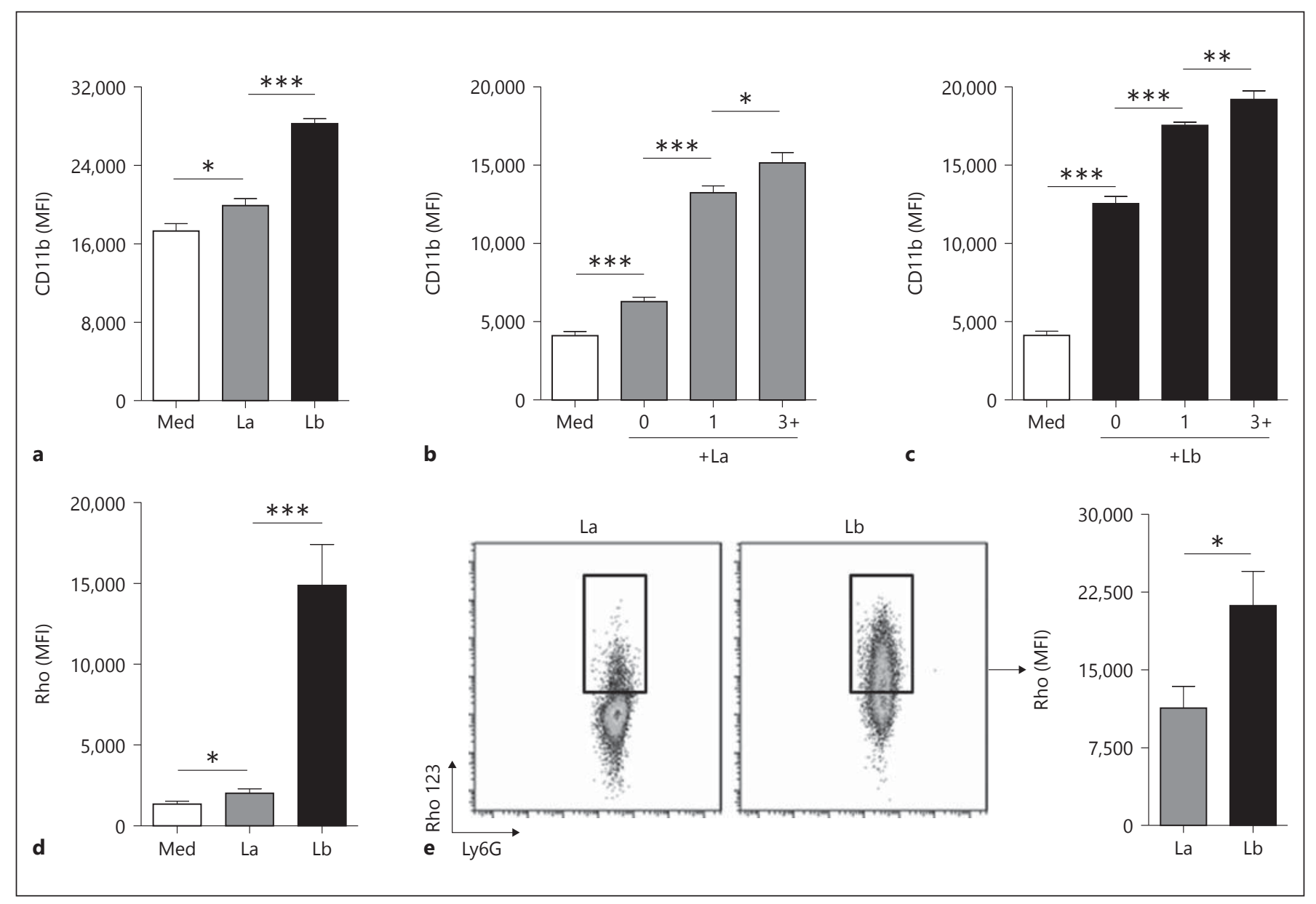

Fig. 2. Amastigote-induced neutrophil activation and oxidative burst. Thioglycollate-induced neutrophils were cocultured with unlabeled $(\mathbf{a}, \mathbf{d}, \mathbf{e})$ or CFSE-labeled amastigotes $(\mathbf{b}, \mathbf{c})$ at a MOI of 5 for $4 \mathrm{~h}$, followed by flow cytometric analysis. a MFI of surface $\mathrm{CD} 11 \mathrm{~b}$ on control neutrophils (Med) and neutrophils exposed to unlabeled amastigotes of L. amazonensis (La) or L. braziliensis $(\mathrm{Lb})$. b, c Imaging flow cytometry data, showing CD11b expression on control neutrophils (Med), neutrophils carrying no parasites (0), 1 parasite or $\geq 3$ parasites, respectively. d Oxidative burst in total Ly6G+ neutrophils in medium alone or in response to amastigotes. e Oxidative burst in Ly6G+ Rho 123+ neutrophils, demonstrating that the intensity of oxidative burst was significantly greater in $L$. braziliensis-infected cells than in L. amazonensis-infected cells. ${ }^{*} \mathrm{p}<0.05,{ }^{* *} \mathrm{p}<0.01,{ }^{* * *} \mathrm{p}<0.001$, between indicated groups.

\section{L. braziliensis Amastigotes Independently Induce Neutrophil Degranulation}

Neutrophils can respond to pathogens by releasing presynthesized antimicrobial components into the phagosome and extracellular space (termed degranulation), and this process can play an important role in both pathogen clearance and tissue damage [15]. Degranulation is commonly assessed by measuring the supernatant levels (or enzyme activity) of established granule components, such as MPO [10] or $\beta$-glucosaminidase [16]. However, these assays require relatively high cell concentrations to achieve appropriate sensitivity, and many commercially available kits are not compatible with samples containing serum.
To overcome these limitations, we developed a flow cytometric method for measuring degranulation by quantifying the MPO loss from the cell interior. We validated this assay by comparing the MPO intensity of control neutrophils (containing 100\% of their MPO in intracellular granules) and neutrophils treated with cytochalasin B and fMLP, a combination of signals that potently induces neutrophil degranulation [10]. As shown in figure 3a, a significant decrease in MPO intensity occurred in cytochalasin B/fMLP-treated cells relative to control neutrophils. Importantly, MPO loss from the interior of treated cells was associated with an increase in corresponding MPO activity in the culture supernatants (see online suppl. fig. 
Fig. 3. Neutrophil degranulation in response to Leishmania amastigotes. a Validation of a FACS-based degranulation assay. Thioglycollate-induced neutrophils were treated with cytochalasin B (CytoB, $5 \mathrm{mg} / \mathrm{ml}$ ) for $10 \mathrm{~min}$ and then with fMLP $(10 \mathrm{mM})$ for $4 \mathrm{~h}$, resulting in a decrease in intracellular MPO staining. The MFI of MPO in treated samples was then compared to that of the controls (Med). $\mathbf{b}$ Neutrophil degranulation in response to amastigotes (MOI of 5) and/or LPS. ${ }^{* * *} \mathrm{p}<$ 0.001 , between indicated groups.

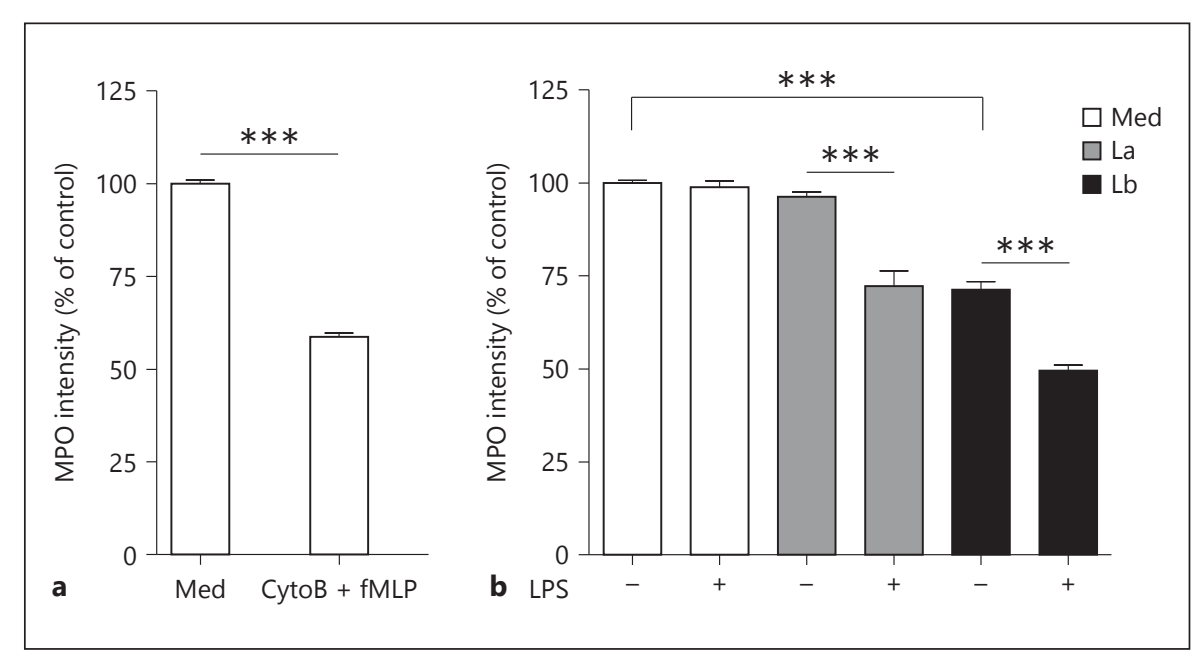

S3A). To quantify amastigote-induced neutrophil degranulation, we examined MPO loss in infected neutrophils after $4 \mathrm{~h}$ of coculture. We observed that L. amazonensis amastigotes were a poor stimulus for degranulation in the absence of a secondary signal, such as LPS (fig. 3b, gray bars). In contrast, L. braziliensis amastigotes induced degranulation independently of a secondary signal, and LPS treatment further enhanced amastigote-induced degranulation (fig. 3b, black bars). We also noted that L. braziliensis metacyclic promastigotes induced a degranulation response similar to that of L. braziliensis amastigotes (online suppl. fig. S3B). These data demonstrate that $L$. amazonensis and L. braziliensis differ in their ability to induce neutrophil degranulation, and that poor L. amazonensismediated degranulation can be amplified in the presence of a secondary proinflammatory signal.

\section{L. braziliensis Amastigotes Are More Susceptible}

to Direct Neutrophil Killing than L. amazonensis

\section{Amastigotes}

Neutrophil activity against promastigotes of certain parasite species is well documented. We and other study groups have previously demonstrated that L. amazonensis promastigotes, but not amastigotes, can be efficiently killed by neutrophils and select neutrophil components, such as NETs and purified histones $[7,8,17]$. Neutrophils also appear to play an important role in limiting parasite burden during the early stages of $L$. braziliensis infection, as coinjection of neutrophils with L. braziliensis promastigotes has been found to significantly reduce parasite burden and strengthen antiparasite immunity [18]. However, L. braziliensis resistance against neutrophil microbicidal activity remains uncharacterized. To determine if amastigote resistance to neutrophil killing is a conserved phenomenon, we added L. amazonensis and L. braziliensis amastigotes to medium alone or to medium containing a surplus of neutrophils (a ratio of 10 neutrophils/1 parasite). After $6 \mathrm{~h}$ of incubation, parasite survival was assessed by limiting dilution assay, as previously described [5]. As shown in figure 4a, coculture of L. braziliensis amastigotes with neutrophils resulted in a significant reduction (approx. 60\%; $\mathrm{p}<0.001$ ) in parasite survival when compared to amastigotes in medium alone. Coculture with PMA-activated neutrophils enhanced the killing of $L$. braziliensis parasites by an additional $15 \%$, while adding DNase to PMA-treated cells partially protected L. braziliensis from neutrophil-mediated killing. In sharp contrast, control or PMA-activated neutrophils were unable to efficiently kill $L$. amazonensis (fig. $4 \mathrm{~b}$ ). These findings highlight distinct differences in the ability of neutrophils to clear different Leishmania sp.

\section{Neutrophils Promote Amastigote Clearance from Infected Macrophages}

Several studies have assessed the ability of neutrophils to reduce Leishmania burden in infected macrophages [18-20]; however, the effect of neutrophils on L. braziliensis amastigote-infected macrophages remains uncharacterized. To explore the interaction between neutrophils and infected macrophages, we loaded bone marrow-derived macrophages with amastigotes and added thioglycollate-induced (control) neutrophils for $24 \mathrm{~h}$. At the end of incubation, we added parasite medium to liberate surviving parasites from the macrophages, and assessed subsequent parasite growth after 3-4 days. We found that adding neutrophils significantly decreased the intramac- 

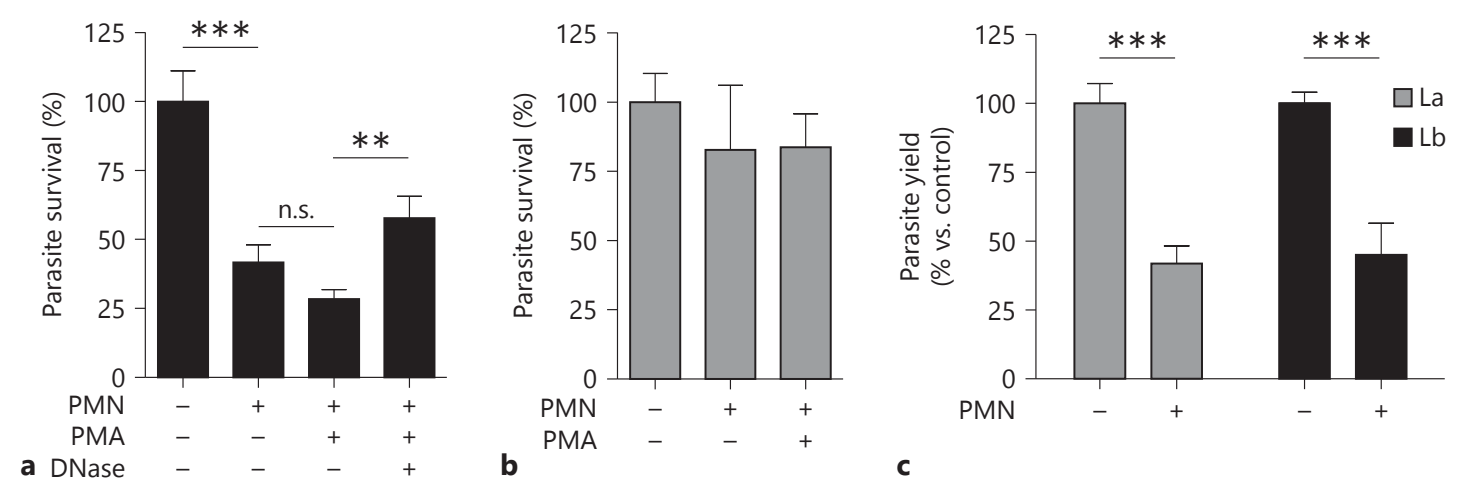

Fig. 4. Neutrophil leishmanicidal activity against L. amazonensis (La) and L. braziliensis (Lb) amastigotes. Thioglycollate-induced neutrophils were cocultured with amastigotes at a ratio of 1 parasite/10 neutrophils (MOI of 0.1 ) for $6 \mathrm{~h}$ in the presence or absence of PMA (100 nM) and/or DNase (100 U/ml). a Reduced survival of $L$. braziliensis amastigotes after coculture with control neutrophils or PMA-activated neutrophils as well as improved parasite survival in PMA-activated wells via treatment with DNase. b Survival of L. amazonensis amastigotes was not significantly altered

rophagic survival of both L. amazonensis and L. braziliensis after $24 \mathrm{~h}$ of coculture (fig. $4 \mathrm{c}$ ). These findings suggest that neutrophils may play an important role in enhancing macrophage antimicrobial activity against multiple species of Leishmania amastigotes.

\section{L. braziliensis Amastigotes Induce More Neutrophil Proinflammatory Cytokine Release than \\ L. amazonensis Amastigotes}

Neutrophil activation is typically accompanied by cytokine release, and we recently reported that L. amazonensis amastigotes and promastigotes preferentially trigger IL-10 and TNF- $\alpha$ release from neutrophils, respectively [7]. To better understand neutrophil interactions with parasites, we characterized cytokine release in response to amastigotes of L. amazonensis and L. braziliensis via multiplex. We found that neutrophils respond to both parasite species by releasing appreciable amounts of several cytokines, including IL-22, TNF- $\alpha$ and IL-18 (fig. 5 a-c). Overall, L. braziliensis amastigotes induced significantly greater levels of all of these cytokines than did L. amazonensis amastigotes. Because there are few reports of IL-22 release from neutrophils [21], we confirmed amastigote-mediated neutrophil IL-22 production via flow cytometry by gating on Ly6G+ CD11b+ cells. We noted that neutrophils exposed to L. braziliensis by the presence of control or PMA-activated neutrophils. c Bone marrow-derived macrophages were infected with amastigotes (MOI of 3) for $24 \mathrm{~h}$ followed by the addition of neutrophils (at a ratio of 3:1 neutrophils/macrophages) for $24 \mathrm{~h}$. Neutrophil-mediated clearance of amastigotes from infected macrophages was measured 3-4 days later via counting (see Materials and Methods). ${ }^{* *} \mathrm{p}<0.01,{ }^{* * *} \mathrm{p}<0.001$, between indicated groups. n.s. $=$ Not significant.

amastigotes began producing detectable amounts of IL22 within $4 \mathrm{~h}$, while early IL-22 production in response to L. amazonensis amastigotes was not significant (fig. 5d). These findings indicate that L. braziliensis amastigotes may induce a rapid and diverse neutrophil cytokine response shortly after cell-parasite interaction.

\section{Discussion}

Multiple species of Leishmania can induce leishmaniasis in humans and animals. However, despite belonging to the same genus, some parasite species have been genetically distinct for 40-80 million years [22]. Therefore, it is highly likely that individual species have developed unique strategies to establish infection in mammalian hosts and maintain chronic persistence. We have previously demonstrated that L. amazonensis and L. braziliensis amastigotes differ in their ability to induce DC activation and cytokine production $[3,4]$. In this study, we examined whether these two parasite species interact with neutrophils differently as well. The rationale of examining amastigote-neutrophil interactions is multifold. Firstly, sand fly- or needle-delivered promastigotes are converted into amastigotes within 1-2 days, when neutrophils are prevalent in the lesions. Secondly, neutrophils are readily 


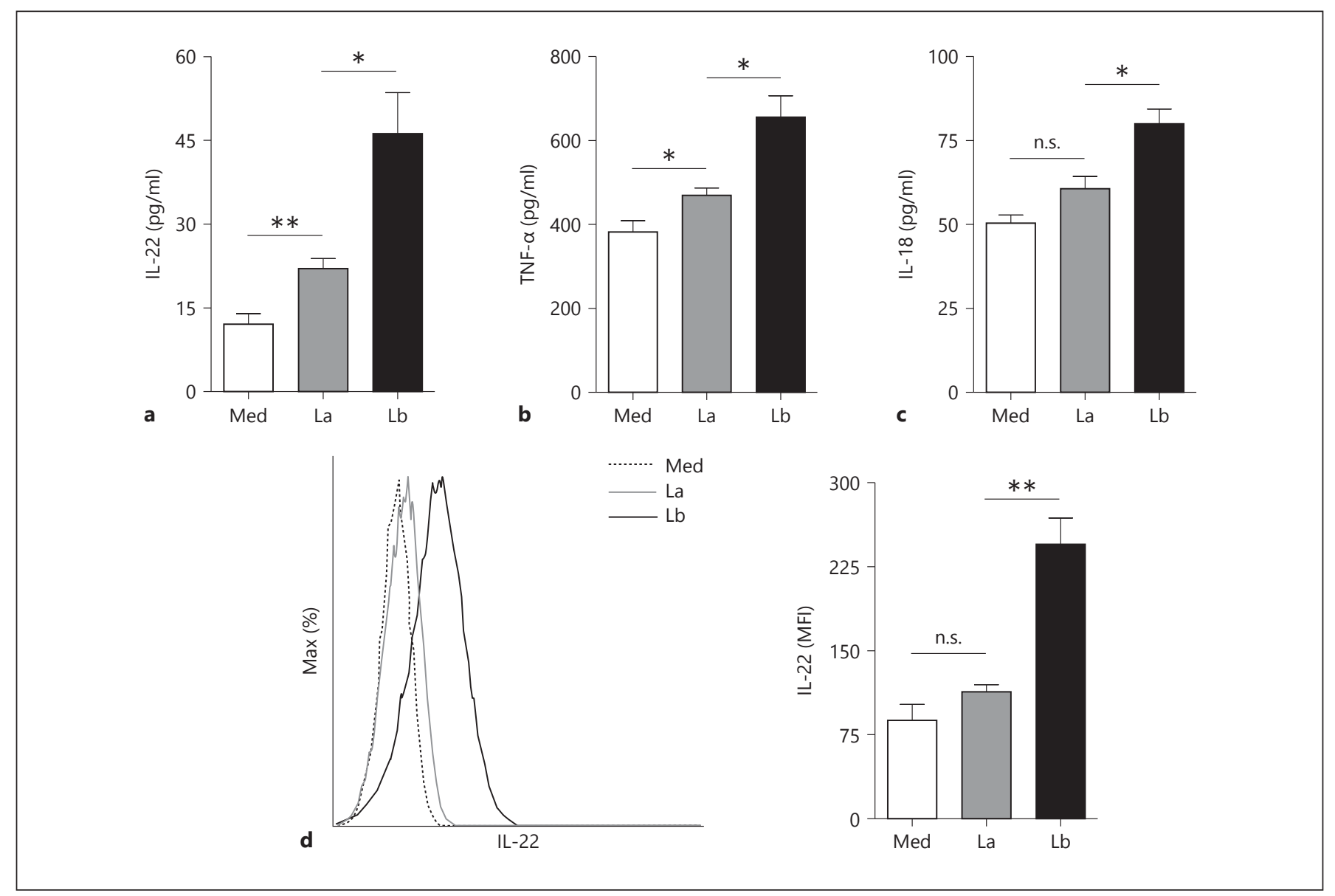

Fig. 5. Amastigote-induced cytokine production. a-c Thioglycollate-induced neutrophils were cocultured with amastigotes of L. amazonensis (La) or L. braziliensis (Lb) at a MOI of 5 for $24 \mathrm{~h}$. The levels of IL-22, TNF- $\alpha$ and IL-18 in culture supernatants were assessed by multiplex. d Neutrophils were cocultured with amastigotes for $4 \mathrm{~h}$ followed by flow cytometric detection of IL-22 in Ly6G+ CD $11 b+$ cells. ${ }^{*} \mathrm{p}<0.05,{ }^{* *} \mathrm{p}<0.01$, between indicated groups. Med $=$ Control neutrophils; n.s. $=$ not significant. detected in chronic forms of cutaneous leishmaniasis in patients infected with $L$. amazonensis, L. mexicana or L. braziliensis [9, 23-25]. At present, there is limited information on how neutrophils interact with these amastigotes. Here, we provide evidence that $L$. braziliensis amastigotes induced significantly more neutrophil activation, oxidative burst, degranulation and cytokine release than did L. amazonensis amastigotes. This was highly associated with direct leishmanicidal activity against L. braziliensis amastigotes, but not $L$. amazonensis amastigotes.

By taking advantage of novel flow-cytometry-based technologies, we were able to perform a detailed, side-byside comparison of neutrophil responses to two distinct Leishmania sp. First, we used imaging flow cytometry to assess the multiplicity of amastigote infection on a percell basis (fig. 1b, c) and demonstrated that neutrophils carrying multiple L. braziliensis parasites exhibited the most activated phenotype (fig. 2c). These data support and expand our observations that L. braziliensis amastigotes are an efficient trigger for neutrophil oxidative burst (fig. 2d, e) and cytokine production (fig. 5a-d). Second, we developed a quantitative FACS-based method for measuring murine neutrophil degranulation, which demonstrated that infection with $L$. braziliensis amastigotes (but not L. amazonensis amastigotes) was sufficient to trigger neutrophil degranulation independently of a secondary proinflammatory signal (fig. $3 \mathrm{~b}$ ). This novel degranulation assay can easily be incorporated into a multicolor flow cytometry panel, which is ideal for the analysis of mixed cell populations (e.g. infected vs. uninfected cells or neutrophil-macrophage cocultures) or for precious samples. 
At this time, it remains unclear why L. braziliensis amastigotes are such a potent stimulus for neutrophil activation, oxidative burst and degranulation but L. amazonensis amastigotes are poor at triggering these responses. We suspect that differences in PAMPs (pathogen-associated molecular patterns) may play an important role in differential recognition of these two parasite species. The importance of PAMPs in parasite recognition was recently explored by Tavares et al. [26], who demonstrated that L. amazonensis internalization and proinflammatory mediator expression by neutrophils were largely driven by interactions between parasite ligands and TLR2. We also suspect that the activation status of neutrophils influences their ability to recognize Leishmania amastigotes. Consequently, it will be interesting to further examine the features of amastigote infection-triggered neutrophil activation via using resting, bone marrow-derived neutrophils.

We have previously discussed the impressive resistance of L. amazonensis amastigotes to neutrophil and macrophage microbicidal activity $[7,27]$ as well as their resistance to direct contact with noxious ROS, nitric oxide and histones $[8,27]$. Currently, it is unclear why L. braziliensis amastigotes are significantly more susceptible to killing by neutrophils than L. amazonensis amastigotes (fig. $4 \mathrm{a}, \mathrm{b}$ ). The findings that $L$. braziliensis amastigote clearance is enhanced in PMA-activated neutrophils and that adding DNase abrogates this effect, suggest that this parasite species may be more susceptible to NETs than are L. amazonensis amastigotes. Although parasiteinduced NET formation has been reported for several Leishmania sp., including L. amazonensis [7, 8, 17], L. braziliensis-mediated NETosis remains a likely but unconfirmed event at this time. Notably, intramacrophagic L. braziliensis and L. amazonensis were cleared with equal efficiency in neutrophil-macrophage cocultures (fig. 4c), indicating that $L$. amazonensis resistance against direct neutrophil microbicidal activity does not protect them from alternative neutrophil-dependent clearance mechanisms. It will be interesting to determine the impact of amastigote-infected macrophages on neutrophil activation, degranulation and cytokine production in future studies.

IL-22 is an IL-10 family member that is tightly linked to neutrophils and Th17-mediated inflammation [28]. When appropriately regulated, IL-22 can contribute to barrier immunity by signaling through nonhematopoietic cells to increase the expression of G-CSF, IL-1 $\beta$, IL-6, CXCL1 and antimicrobial peptides such as defensins [28]. However, sustained IL-22 production may also play a pathogenic role in chronic or highly dysregulated in- flammatory foci, such as those found in psoriasis [29] and rheumatoid arthritis [30]. Curiously, reports of IL-22 involvement in Leishmania control and pathogenesis are sparse. Hezarjaribi et al. [31] demonstrated that exogenous IL-22 enhanced the protective effects of vaccination in $\mathrm{BALB} / \mathrm{c}$ mice infected with $L$. major, resulting in increased IFN- $\gamma$ and reduced IL-4 expression. In addition, Pitta et al. [32] demonstrated that Leishmania antigeninduced IL-22 production in human peripheral blood mononuclear cells correlates with protection against visceral leishmaniasis. Given that mucocutaneous leishmaniasis in humans is associated with a highly dysregulated Th17-like response and persistent neutrophil recruitment [9], it is tempting to speculate that IL-22 plays an important role in maintaining granulocyte recruitment and perpetuating tissue damage in this disease manifestation. At this stage, it is unclear how other neutrophil-derived cytokines (e.g. TNF- $\alpha$ and IL-18) interact with IL-22 to facilitate parasite clearance. We believe that additional studies are warranted to quantify IL-22 production in the lesions of cutaneous and mucocutaneous leishmaniasis patients and to further investigate the role of neutrophils and IL-22 in animal models of L. braziliensis infection.

Antiparasite immunity is a complex process involving multiple innate and adaptive immune cell types, and the concerted effort of these cells influences the balance between parasite clearance and inflammatory pathology. This study has helped to identify novel cellular and molecular mechanisms that drive differential immune recognition and control of New World Leishmania spp., and highlights a potential role for neutrophils in this intricate process. The capacity for neutrophils to kill amastigotes (either directly or indirectly) and to influence the surrounding inflammatory milieu should be important considerations for future studies.

\section{Acknowledgements}

This study was supported by an NIH grant (R56AI043003) and the UTMB IHII pilot grant to L. Soong. E.D. Carlsen was supported by an NIH predoctoral training grant T32AI007526 (PI: A. Barrett), the UTMB McLaughlin Endowment predoctoral fellowship and the Blocker Scholar Fellowship in Biomedical Research. C.A. Henard was supported by an NIH postdoctoral training grant (T32AI00753613) (PI: C. White, Jr.). C. Hay was supported by the UTMB McLaughlin Endowment predoctoral fellowship. H. de Matos Guedes was supported by the ASTMH Gorgas Memorial Institute Research Award and a CAPES postdoctoral fellowship. We thank Mardelle Susman for assisting in manuscript preparation. 


\section{References}

-1 Paniz Mondolfi AE, Duffey GB, Horton LE, Tirado M, Reyes Jaimes O, Perez-Alvarez A, Zerpa O: Intermediate/borderline disseminated cutaneous leishmaniasis. Int J Dermatol 2013;52:446-455.

2 Soong L: Subversion and utilization of host innate defense by Leishmania amazonensis. Front Immunol 2012;3:58.

3 Vargas-Inchaustegui DA, Xin L, Soong L: Leishmania braziliensis infection induces dendritic cell activation, ISG15 transcription, and the generation of protective immune responses. J Immunol 2008; 180:7537-7545.

$\checkmark 4$ Xin L, Li K, Soong L: Down-regulation of dendritic cell signaling pathways by Leishmania amazonensis amastigotes. Mol Immunol 2008;45:3371-3382.

5 Novais FO, Nguyen BT, Beiting DP, Carvalho LP, Glennie ND, Passos S, Carvalho EM, Scott $P$ : Human classical monocytes control the intracellular stage of Leishmania braziliensis by reactive oxygen species. J Infect Dis 2014;209: 1288-1296.

6 Passwell JH, Shor R, Smolen J, Jaffe CL: Infection of human monocytes by Leishmania results in a defective oxidative burst. Int J Exp Pathol 1994;75:277-284.

7 Carlsen ED, Hay C, Henard CA, Popov V, Garg NJ, Soong L: Leishmania amazonensis amastigotes trigger neutrophil activation but resist neutrophil microbicidal mechanisms. Infect Immun 2013;81:3966-3974.

8 Wang Y, Chen Y, Xin L, Beverley SM, Carlsen ED, Popov V, Chang KP, Wang M, Soong L: Differential microbicidal effects of human histone proteins $\mathrm{H} 2 \mathrm{~A}$ and $\mathrm{H} 2 \mathrm{~B}$ on Leishmania promastigotes and amastigotes. Infect Immun 2011;79:1124-1133.

-9 Boaventura VS, Santos CS, Cardoso CR, de Andrade J, Dos Santos WL, Clarencio J, Silva JS, Borges VM, Barral-Netto M, Brodskyn CI, Barral A: Human mucosal leishmaniasis: neutrophils infiltrate areas of tissue damage that express high levels of Th17-related cytokines. Eur J Immunol 2010;40:2830-2836.

$\checkmark 10$ Abdel-Latif D, Steward M, Macdonald DL, Francis GA, Dinauer MC, Lacy P: Rac2 is critical for neutrophil primary granule exocytosis. Blood 2004;104:832-839.

11 Andrews PC, Krinsky NI: Quantitative determination of myeloperoxidase using tetramethylbenzidine as substrate. Anal Biochem 1982;127:346-350.

12 Schymeinsky J, Mocsai A, Walzog B: Neutrophil activation via beta2 integrins (CD11/
CD18): molecular mechanisms and clinical implications. Thromb Haemost 2007;98:262-273.

13 Sorensen NB, Nielsen HL, Varming K, Nielsen H: Neutrophil activation by Campylobacter concisus. Gut Pathog 2013;5:17.

14 Rothe G, Emmendorffer A, Oser A, Roesler J, Valet G: Flow cytometric measurement of the respiratory burst activity of phagocytes using dihydrorhodamine 123. J Immunol Methods 1991;138:133-135.

15 Klebanoff SJ: Myeloperoxidase: friend and foe. J Leukoc Biol 2005;77:598-625.

16 Sato T, Hongu T, Sakamoto M, Funakoshi Y, Kanaho Y: Molecular mechanisms of $\mathrm{N}$-formyl-methionyl-leucyl-phenylalanine-induced superoxide generation and degranulation in mouse neutrophils: phospholipase D is dispensable. Mol Cell Biol 2013;33:136145.

17 Guimaraes-Costa AB, Nascimento MT, Froment GS, Soares RP, Morgado FN, Conceicao-Silva F, Saraiva EM: Leishmania amazonensis promastigotes induce and are killed by neutrophil extracellular traps. Proc Natl Acad Sci U S A 2009;106:6748-6753.

18 Novais FO, Santiago RC, Bafica A, Khouri R, Afonso L, Borges VM, Brodskyn C, BarralNetto M, Barral A, de Oliveira CI: Neutrophils and macrophages cooperate in host resistance against Leishmania braziliensis infection. J Immunol 2009;183:8088-8098.

19 Ribeiro-Gomes FL, Otero AC, Gomes NA, Moniz-De-Souza MC, Cysne-Finkelstein L, Arnholdt AC, Calich VL, Coutinho SG, Lopes MF, DosReis GA: Macrophage interactions with neutrophils regulate Leishmania major infection. J Immunol 2004;172:4454-4462.

20 de Souza Carmo EV, Katz S, Barbieri CL: Neutrophils reduce the parasite burden in Leishmania (Leishmania) amazonensis-infected macrophages. PLoS One 2010;5: e13815.

21 Zindl CL, Lai JF, Lee YK, Maynard CL, Harbour SN, Ouyang W, Chaplin DD, Weaver CT: IL-22-producing neutrophils contribute to antimicrobial defense and restitution of colonic epithelial integrity during colitis. Proc Natl Acad Sci U S A 2013;110:12768-12773.

22 McMahon-Pratt D, Alexander J: Does the Leishmania major paradigm of pathogenesis and protection hold for New World cutaneous leishmaniases or the visceral disease? Immunol Rev 2004;201:206-224.

23 Dabiri S, Hayes MM, Meymandi SS, Basiri M, Soleimani F, Mousavi MR: Cytologic features of 'dry-type' cutaneous leishmaniasis. Diagn Cytopathol 1998;19:182-185.

24 Daboul MW: Role of neutrophils in cutaneous leishmaniasis. East Mediterr Health J 2010;16:1055-1058.

25 Dantas ML, Oliveira JM, Carvalho L, Passos ST, Queiroz A, Guimaraes LH, Machado P, Carvalho E, Arruda S: Comparative analysis of the tissue inflammatory response in human cutaneous and disseminated leishmaniasis. Mem Inst Oswaldo Cruz 2014;109:202209.

26 Tavares NM, Araujo-Santos T, Afonso L, Nogueira PM, Lopes UG, Soares RP, Bozza PT, Bandeira-Melo C, Borges VM, Brodskyn C: Understanding the mechanisms controlling Leishmania amazonensis infection in vitro: the role of LTB4 derived from human neutrophils. J Infect Dis 2014;210:656-666.

27 Henard CA, Carlsen ED, Hay C, Kima PE, Soong L: Leishmania amazonensis amastigotes highly express a tryparedoxin peroxidase isoform that increases parasite resistance to macrophage antimicrobial defenses and fosters parasite virulence. PLoS Negl Trop Dis 2014;8:e3000.

28 Rutz S, Eidenschenk C, Ouyang W: IL-22, not simply a Th17 cytokine. Immunol Rev 2013; 252:116-132.

29 Sa SM, Valdez PA, Wu J, Jung K, Zhong F, Hall L, Kasman I, Winer J, Modrusan Z, Danilenko DM, Ouyang W: The effects of IL20 subfamily cytokines on reconstituted human epidermis suggest potential roles in cutaneous innate defense and pathogenic adaptive immunity in psoriasis. J Immunol 2007; 178:2229-2240.

30 Geboes L, Dumoutier L, Kelchtermans H, Schurgers E, Mitera T, Renauld JC, Matthys P: Proinflammatory role of the Th17 cytokine interleukin-22 in collagen-induced arthritis in C57BL/6 mice. Arthritis Rheum 2009;60: 390-395.

31 Hezarjaribi HZ, Ghaffarifar F, Dalimi A, Sharifi Z, Jorjani O: Effect of IL-22 on DNA vaccine encoding LACK gene of Leishmania major in BALB/c mice. Exp Parasitol 2013; 134:341-348.

32 Pitta MG, Romano A, Cabantous S, Henri S, Hammad A, Kouriba B, Argiro L, el Kheir M, Bucheton B, Mary C, El-Safi SH, Dessein A: IL-17 and IL-22 are associated with protection against human kala azar caused by Leishmania donovani. J Clin Invest 2009;119: 2379-2387.
Neutrophil Leishmanicidal Activity against $L$. braziliensis
J Innate Immun 2015;7:354-363 DOI: $10.1159 / 000373923$ 